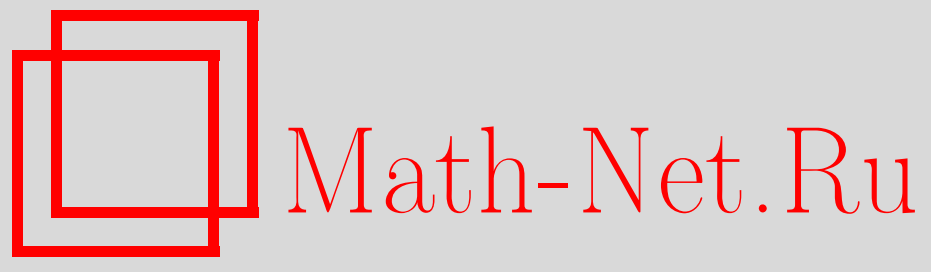

М. И. Штогрин, Специальные изометрические преобразования конуса, УМH, 2008, том 63, выпуск 2, 183-184 DOI: https://doi.org/10.4213/rm9188

Использование Общероссийского математического портала Math-Net.Ru подразумевает, что вы прочитали и согласны с пользовательским соглашением http://www . mathnet.ru/rus/agreement

Параметры загрузки:

IP: 18.208 .226 .222

26 апреля 2023 г., 15:52:57

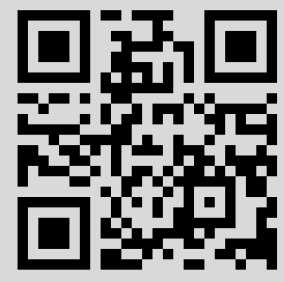




\title{
Специальные изометрические преобразования конуса
}

\author{
М. И. Штогрин
}

Исследуются специальные изометрические вложения поверхности прямого кругового конуса в пространство $\mathbb{R}^{3}$. Строение вложенной поверхности обладает двоякой периодичностью. В окружном направлении действует группа $n m m$ порядка $2 n$ (порожденная отражениями от двух плоскостей, составляющих угол $\pi / n)$. Вдоль оси действует дискретная группа гомотетий с центром в вершине конуса. Периодом последней может служить боковая поверхность усеченной правильной пирамиды.

Возьмем вспомогательную усеченную правильную пирамиду П с горизонтальным $2 n$-угольным основанием и вертикальной осъю. На боковой грани $\alpha_{1}$ возьмем произвольную гладкую кривую $\gamma_{1}$, однозначно проектирующуюся на ось. На смежной боковой грани $\alpha_{2}$ построим кривую $\gamma_{2}$, получающуюся из $\gamma_{1}$ при зеркальном отражении от плоскости $\beta$, проходящей через боковое ребро $\alpha_{2} \cap \alpha_{1}$ и ось. Аналогично строим кривую $\gamma_{3}$ на грани $\alpha_{3}$, смежной с $\alpha_{2}$, и т. д. Так на каждой грани $\alpha_{i}$ получим кривую $\gamma_{i}$. Проведем теперь через кривые $\gamma_{1}$ и $\gamma_{2}$ цилиндрическую поверхность $Z_{12}$ с образующими, перпендикулярными плоскости $\beta$. Аналогично строим цилиндрические поверхности $Z_{23}, Z_{34}$ и т. д. Все вместе они составляют кусочно гладкую поверхность $Z$ с ребристыми линиями $\gamma_{i}, i=1, \ldots, 2 n$.

Именно так строил А.В. Погорелов [1] оригинальную трубчатую поверхность $Z$ в том случае, когда в качестве П была взята призма, а не пирамида.

Если же П является пирамидой, то поверхность $Z$, вообе говоря, не развертывающаяся: никакая достаточно малая окрестность точки ребристой линии $\gamma_{i}$ не изометрична евклидову кругу. Однако, если П есть пирамида, а $\gamma_{i}$ суть прямолинейные отрезки, обозначим их через $\ell_{i}$, то поверхность $Z$ является кусочно линейной. Обозначим ее через $\bar{Z}$. Любая точка ребра $\ell_{i}$ имеет окрестность, состоящую из двух полукругов. Поверхность $\bar{Z}$ развертывающаяся. Она ворончатая.

Кусочно линейная поверхность $\bar{Z}$ с прямолинейными ребрами $\ell_{i}$, взятыми на гранях $\alpha_{i}$ усеченной правильной пирамиды П, допускает кусочно гладкие изометрические вложения в трехмерное евклидово пространство $\mathbb{R}^{3}$. Предъявим их.

Если $\ell_{1}$ есть ось симметрии грани $\alpha_{1}$, то поверхность $\bar{Z}$ представляет собой усеченную правильную пирамиду. Мы рассмотрим более общий случай.

$\mathrm{K}$ краям кусочно линейной поверхности $\bar{Z}$ приклеим два выпуклых многоугольника. Получим замкнутый выпуклый многогранник. Тут $\ell_{j} \cap \ell_{i}=\emptyset, j \neq i$. В вершине нижнего основания сходятся три плоских угла $\varphi, \psi$ и $\pi-\pi / n$. В вершине верхнего основания сходятся три плоских угла $\pi-\varphi, \pi-\psi$ и $\pi-\pi / n$. Те и эти три угла удовлетворяют неравенствам треугольника. Сумма плоских углов в вершине многогранника меньше $2 \pi$. Поэтому углы $\varphi$ и $\psi$ удовлетворяют следующим системам неравенств:

$$
\begin{gathered}
\varphi<\psi+\pi-\frac{\pi}{n}, \quad \psi<\varphi+\pi-\frac{\pi}{n} ; \\
\pi-\frac{\pi}{n}<\varphi+\psi<\pi+\frac{\pi}{n} .
\end{gathered}
$$

Все плоские куски $\bar{Z}_{12}, \ldots, \bar{Z}_{2 n 1}$ кусочно линейной ворончатой поверхности $\bar{Z} \subset \mathbb{R}^{3}$ суть грани. Разрезав грань $\bar{Z}_{2 n 1}$ одной из $n$ плоскостей зеркальной симметрии, развернем поверхность $\bar{Z}$ на евклидову плоскость $\mathbb{R}^{2}$. Получим развертку поверхности $\bar{Z}$ в виде невыпуклого (из-за пирамиды П) многоугольника $R \subset \mathbb{R}^{2}$, обладающего парой эквивалентных сторон, которые имеют один и тот же образ на поверхности $\bar{Z}$ при изометрическом отображении $\bar{f}: R \rightarrow \bar{Z}$. Отображение $\bar{f}$ сохраняет углы.

Работа выполнена при финансовой поддержке РФФИ (грант 08-01-90102), программы "Ведущие научные школы" (грант НШ-4182.2006.1) и государственной программы фундаментальных исследований ОМН РАН “Современные проблемы теоретической математики”. 
Развертка наследует условия $(*)$ и $(* *)$, где $\varphi$ и $\psi$ суть углы, составляемые прообразом ребра $\ell_{1}$ в одном из его концов, скажем, $A$, со сторонами развертки $R$. Прообразы следов имеющихся $n$ плоскостей зеркальной симметрии на поверхности $\bar{Z}$ суть отрезки на плоскости $\mathbb{R}^{2}$, которые при $\ell_{j} \cap \ell_{i}=\emptyset, j \neq i$, разбивают развертку $R$ на $2 n$ невыпуклых фундаменталъных шестиугольников - прообразов фундаментальных областей группы $n m m$. Прообразы отрезков $\ell_{i}$, которые изначально были взяты на гранях $\alpha_{i}$ пирамиды П, расположенные в смежных шестиугольниках развертки $R$, зеркально симметричны относительно их общей стороны.

Пусть кусочно линейная поверхность $\bar{Z} \subset \mathbb{R}^{3}$ метрически задана. Тогда ее развертка $R \subset \mathbb{R}^{2}$ метрически задана. Задан ее угол $\angle A$. Для него имеет место равенство $\angle A=\varphi+\psi=$ const. Для слагаемых $\varphi$ и $\psi$ выполнена система неравенств $(* *)$.

Возьмем произвольную гладкую кривую $\tau_{1}$, все внутренние точки которой расположены внутри того же шестиугольника развертки $R$, что и точки прообраза ребра $\ell_{1}$, и которая обладает общими концами с прообразом ребра $\ell_{1}$. Обозначим через $\varphi^{*}$ и $\psi^{*}$ углы, которые составляет касательная в произвольной точке кривой $\tau_{1}$ со сторонами развертки $R$, проходящими через ее вершину $A$. Выполнение неравенств $(* *)$ для углов $\varphi^{*}$ и $\psi^{*}$ следует из $\varphi^{*}+\psi^{*}=\angle A=\varphi+\psi$. Наше главное требование - в каждой точке кривой $\tau_{1}$ углы $\varphi^{*}$ и $\psi^{*}$ удовлетворяют неравенствам $(*)$. Далее, во всех фундаментальных шестиугольниках развертки $R$ возьмем гладкие кривые $\tau_{i}$, конгруэнтные гладкой кривой $\tau_{1}$, пользуясь единственным правилом: в смежных шестиугольниках гладкие кривые $\tau_{i}$ зеркально симметричны относительно их общей стороны. Теперь аналогично [2] строим изометрическое вложение развертки $f^{*}: R \rightarrow Z^{*}$, где $Z^{*} \subset \mathbb{R}^{3}$.

Теорема. Кусочно линейная ворончатая поверхность $\bar{Z}$, построенная с помощью прямолинейного ребра $\ell_{1}$, взятого на грани $\alpha_{1}$ пирамиды П, допускает изометрическое вложение в трехмерное евклидово пространство $\mathbb{R}^{3}$ в виде кусочно гладкой ворончатой поверхности $Z^{*}$, состоящей из $2 n$ иилиндрических поверхностей и обладающей $2 n$ конгруэнтными криволинейными ${ }^{1}$ ребрами $\gamma_{i}^{*}$, прообразами которых являются построенные выше гладкие кривые $\tau_{i}$, удовлетворяющие неравенствам (*).

Рассмотрим специальную вариацию прообраза ребра $\ell_{1}$ на развертке $R$. Будем предполагать, что варьируемая кривая, обозначим ее $\tau_{1}$, зависит от параметра $t$, $0 \leqslant t \leqslant 1$. В процессе вариации выполняются следующие условия: варьируемая кривая $\tau_{1}$ находится внутри того же шестиугольника, что и прообраз ребра $\ell_{1}$; концы варьируемой кривой $\tau_{1}$ остаются неподвижными - они совпадают с концами прообраза ребра $\ell_{1}$; варьируемая кривая $\tau_{1}$ остается гладкой; углы $\varphi^{*}$ и $\psi^{*}$, которые составляет касательная к варьируемой кривой $\tau_{1}$ в любой ее точке со сторонами развертки $R$, проходящими через вершину $A$, удовлетворяют неравенствам $(*)$. Далее, во всех шестиугольниках развертки $R$ построим варьируемые гладкие кривые $\tau_{i}, i=1, \ldots, 2 n$, конгруэнтные варьируемой гладкой кривой $\tau_{1}$, пользуясь единственным правилом: при каждом фиксированном $t, 0 \leqslant t \leqslant 1$, в смежных шестиугольниках развертки $R$ кривые $\tau_{i}$ зеркально симметричны относительно их общей стороны.

СледСтвиЕ. При указанных выше условиях любая вариация прообраза ребра $\ell_{1}$ сопровождается вариачией кривой $\gamma_{1}^{*}$, задающей изгибание поверхности $Z^{*}$.

\section{Список литературы}

[1] А. В. Погорелов, Изгибания поверхностей и устойчивость оболочек, Наука, М., 1986; 2-е изд., Наукова думка, Киев, 1998. [2] М. И. Штогрин, УМН, $62: 5$ (2007), 173-174.

М. И. Штогрин (M. I. Shtogrin)

Математический институт им. В. А. Стеклова РАН

E-mail: stogrin@mi.ras.ru
Представлено В. М. Бухштабером Принято редколлегией 10.02 .2008

\footnotetext{
${ }^{1}$ Ребра $\gamma_{i}^{*}$ являются пространственными кривыми, обладающими ненулевым кручением.
} 